\title{
Correlation Between Hot-Carrier-Induced Interface States and GIDL Current Increase in N-MOSFET's
}

\author{
P. T. Lai, J. P. Xu, W. M. Wong, H. B. Lo, and Y. C. Cheng, Member, IEEE
}

\begin{abstract}
Correlation between created interface states and GIDL current increase in n-MOSFET's during hot-carrier stress is quantitatively discussed. A trap-assisted two-step tunneling model is used to relate the increased interface-state density $\left(\Delta D_{\mathrm{it}}\right)$ with the shift in GIDL current $\left(\Delta I_{d}\right)$. Results show that under appropriate drain-gate biases, the two-step tunneling is so dominant that $\Delta I_{d}$ is insensitive to temperatures up to about $50{ }^{\circ} \mathrm{C}$. With the help of 2-D device simulation, the locations of the drain region with significant two-step tunneling and the energy levels of the traps involved can be found, with both depending on the drain voltage. From these insights on $\Delta D_{\mathrm{it}}, \Delta I_{d}$ and their relation, $\Delta D_{\text {it }}$ near the midgap can be estimated, with an error less than $10 \%$ as compared to the results of chargepumping measurement on the same transistors. Devices with nitrided gate oxide, different gate-oxide thicknesses and different channel dimensions are also tested to verify the above correlation.
\end{abstract}

\section{INTRODUCTION}

$\mathbf{I}$ NTERFACE-STATE creation due to hot-carrier stress constitutes a major device-reliability concern and attracts much research interest [1]-[3]. It is generally characterized by charge- pumping (CP) and $C-V$ techniques. However, since its effects on the electrical characteristics of MOSFET's are obvious in terms of subthreshold slope and gate-induced-drainleakage (GIDL) current, it should also be possible to determine the change in interface-trap density $\Delta D_{\mathrm{it}}$ by measuring the shift in these device parameters, which has been described in the literature [4]-[7]. It was suggested by Duvvury et al. [4] that the increase in GIDL current was a direct result of flat-band voltage shift due to $\Delta D_{\text {it. }}$. However, Chen et al. [5] reported that in an $\mathrm{n}^{+}$gated diode, $\Delta D_{\mathrm{it}}$ merely enhances GIDL current via an increase in surface generation velocity. More recently, Hori [6] discussed $\Delta D_{\mathrm{it}}$-related GIDL current based on band-to-band (B-B) tunneling process via interface state. The present work attempts to quantitatively study the correlation between $\Delta D_{\mathrm{it}}$ and GIDL current increase $\left(\Delta I_{d}\right)$ by means of an interface-trap-assisted two-step tunneling model [7], thus obtaining some insights on the physical mechanisms involved.

Manuscript received March 12, 1997; revised August 11, 1997. The review of this paper was arranged by Editor D. P. Verret. This work was supported in part by the RGC and CRCG Research Grants, the University of Hong Kong.

P. T. Lai, W. M. Wong, H. B. Lo, and Y. C. Cheng are with the Department of Electrical and Electronic Engineering, the University of Hong Kong, Hong Kong.

J. P. Xu is with the Department of Solid State Electronics, Huazhong University of Science and Technology, Wuhan, 430074, P.R.C.

Publisher Item Identifier S 0018-9383(98)00954-X.

\section{EXPERIMENT}

Conventional n-MOSFET's with thermal $\mathrm{SiO}_{2}(\mathrm{OX})$ grown at $850{ }^{\circ} \mathrm{C}$ for 100 and $60 \mathrm{~min}$, respectively, in $\mathrm{O}_{2}$ and $\mathrm{Ar}$ (marked as OX1 and OX2, respectively), or nitrided oxide $(\mathrm{N} 2 \mathrm{ON})$ as gate dielectric were used. N2ON samples were fabricated by nitridizing OX2 samples at $950{ }^{\circ} \mathrm{C}$ for $70 \mathrm{~min}$ in pure $\mathrm{N}_{2} \mathrm{O}$ ambient. The final gate-oxide thickness $T_{\mathrm{ox}}$ of the three kinds of samples (measured by $C-V$ ) was 210 $\AA$ for OX1 samples and N2ON samples, and $145 \AA$ for OX2 samples. The devices were subject to hot-electron stress, $V_{G}=0.5 V_{D}=3.5 \mathrm{~V}$, for $1000-4000 \mathrm{~s}$ to create interface traps. GIDL current was measured before and after the stress under three different conditions with the same drain-to-gate bias $V_{\mathrm{DG}}=8 \mathrm{~V}\left(V_{D}=5 \mathrm{~V}, V_{G}=-3 \mathrm{~V} ; V_{D}=4 \mathrm{~V}\right.$, $V_{G}=-4 \mathrm{~V}$; and $\left.V_{D}=3 \mathrm{~V}, V_{G}=-5 \mathrm{~V}\right)$, and source and substrate grounded. The stressings and measurements were performed on MOSFET's with channel length $(L) /$ channel width $(W)=1 / 24 \mu \mathrm{m}$ and $1.8 / 10 \mu \mathrm{m}$ in a nitrogen ambient under light-tight and electrically shielded condition using an HP 4156A precision semiconductor parameter analyzer.

\section{RESUlTS AND DisCUSSION}

Fig. 1 shows the measured pre-stress and post-stress GIDL currents for OX1 samples. Solid lines, dash lines and dot lines correspond to the three different conditions for GIDL measurement. Along the arrow direction, stress time increases from $0 \mathrm{~s}$ to $4000 \mathrm{~s}$ with an increment of $1000 \mathrm{~s}$. $X$-axis is the time at which GIDL current is measured after the stress is finished. It can be seen that after stressing for $1000 \mathrm{~s}$, GIDL current increases obviously, and for stress time above $1000 \mathrm{~s}$, shift of GIDL current becomes gradually small and then reaches almost a saturation value after stressing for $4000 \mathrm{~s}$. This saturation phenomenon seems to indicate that interface-trap creation is limited by the number of potential trap sites available. The observed increase in post-stress GIDL current is distinctly different from that induced by oxide traps because oxide traps only induce GIDL current transient on a time scale of seconds. So, it should be dependent on the amount of interface traps created during stress since the stress method of $V_{G}=0.5 V_{D}$ is mostly responsible for interfacestate generation [1]. In other words, an additional conduction mechanism involving interface traps [7] should be possible after the hot-electron stress. Under low $V_{\mathrm{DG}}$, the energy band is not sufficiently bent with the Fermi level $E_{F}$ still inside the bandgap, and therefore direct B-B tunneling is weak. However, the thermal excitation of electrons from the valence band into the interface traps, followed by tunneling into the conduction 


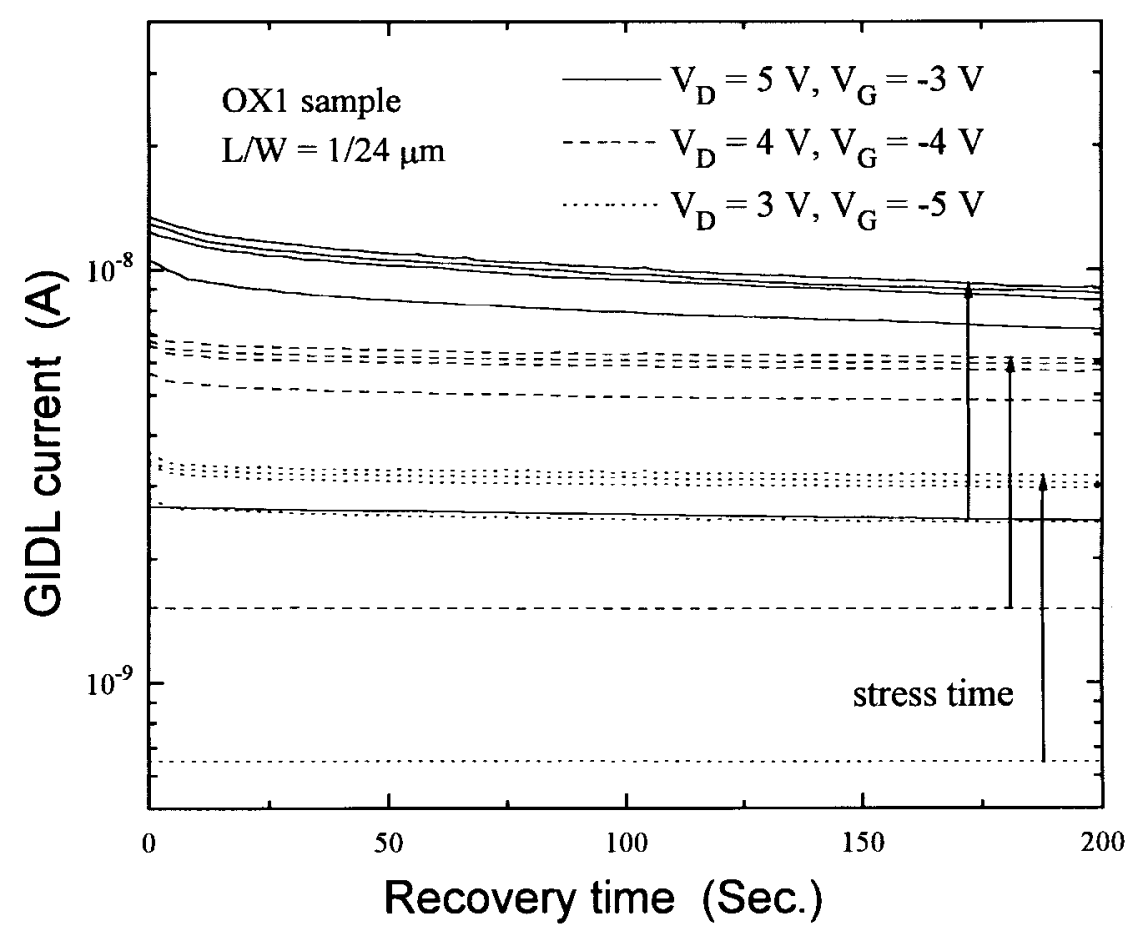

Fig. 1. Measured pre-stress and post-stress GIDL currents for OX1 sample. Stress time is 1000, 2000, 3000, and 4000 s, respectively.

band, i.e., a trap-assisted one-step tunneling [6], is highly probable. As $V_{\mathrm{DG}}$ is increased to such a large value that $E_{F}$ is much below the interface-trap levels, a trap-assisted two-step tunneling is also appreciable due to enhanced tunneling rate, with hole tunneling from the interface traps to the valence band (step 1) and then electron tunneling from the interface traps to the conduction band (step 2) [7], as illustrated in Fig. 2 . This deduction is further supported by the measured results for N2ON sample before and after a stressing for $3000 \mathrm{~s}$, as presented in Fig. 3. It can be seen that shift in post-stress GIDL current is significantly smaller as compared to OX1 sample (only $167 \mathrm{pA}$ for $V_{D}=5 \mathrm{~V}, 105 \mathrm{pA}$ for $V_{D}=4 \mathrm{~V}$ and $40 \mathrm{pA}$ for $V_{D}=3 \mathrm{~V}$ ). This smaller shift is expected because creation of interface states during stress is effectively suppressed due to nitrogen incorporation at the $\mathrm{Si} / \mathrm{SiO}_{2}$ interface of nitrided devices [8]. Thus it can be concluded that the increase of GIDL current after stress indeed reflects a generation of interface states and $\Delta D_{\mathrm{it}}$ could be linked to the measured increase in post-stress GIDL current.

Under the two-step tunneling condition, the increased GIDL current $\Delta I_{d}$ can be derived as follows [7]:

$$
\begin{aligned}
\Delta I_{d} & =A \exp \left(-B_{\mathrm{it}} / F\right) \\
B_{\mathrm{it}} & =\frac{4}{\hbar}\left(2 m_{n}\right)^{1 / 2} \frac{\left(E_{c}-E_{t}\right)^{3 / 2}}{3 q} \\
E_{t} & =\frac{E_{V}+\left(F_{1} / F\right)^{2 / 3} E_{C}}{1+\left(F_{1} / F\right)^{2 / 3}}
\end{aligned}
$$

where $A$ is weakly dependent on electric field and proportional to the generated interface-state density near midgap $\Delta D_{\mathrm{it}}, F_{1}$ is the lateral field, $F$ is the total field in deep-depletion region, and $E_{t}$ is the energy level of the interface traps, which are most effective in the two-step tunneling process. Other variables

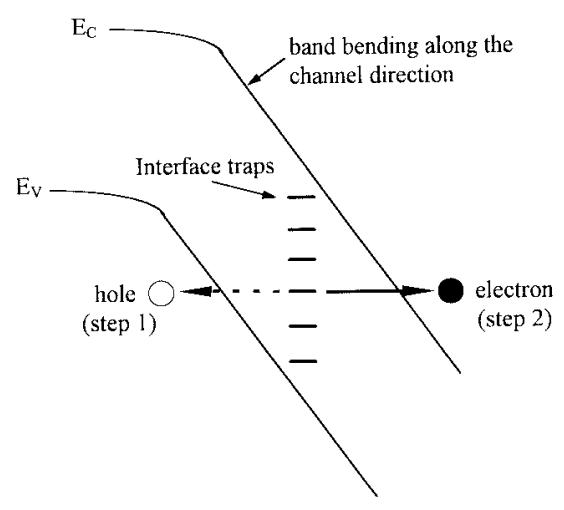

Fig. 2. Schematic diagram of the trap-assisted two-step tunneling, with hole tunneling occurred only in the direction parallel to the channel while electron tunneling occurred in both directions parallel and vertical (band bending is not plotted here) to the channel.

have their usual definitions. To check the validity of (1), the temperature dependence of $\Delta I_{d}$ was measured and the results are shown in Fig. 4. It can be seen that measured $\Delta I_{d}$ at $V_{\mathrm{DG}}=8 \mathrm{~V}$ hardly changes with temperature $(T)$ below $50^{\circ} \mathrm{C}$, but increases at higher temperatures due to thermal generation which is ignored in (1) [7]. From (2) and (3), changes of $\left(E_{t}-E_{V}\right)$ and $B_{\text {it }}$ with $F_{1} / F$ can be obtained, as shown in Fig. 5. It can be seen that as lateral field $F_{1}$ gets stronger, $E_{t}$ changes from $E_{V}$ to $E_{i}$ (intrinsic Fermi level) and the corresponding $B_{\text {it }}$ decreases from 36 to $12.8 \mathrm{MV} / \mathrm{cm}$. In fact, $B_{\text {it }}$ in (1) reflects a potential barrier height in tunneling and has a minimum value for midgap-trap-assisted two-step tunneling. In [7], the parameter $A$ was treated as a fitting parameter, and as a result, $\Delta D_{\text {it }}$ was not explicitly involved in (1). In reality, $A$ can be expressed as

$$
A=\Delta D_{\mathrm{it}} q W L_{e} \Delta E_{t} /\left(2 \tau_{0 c}\right)
$$




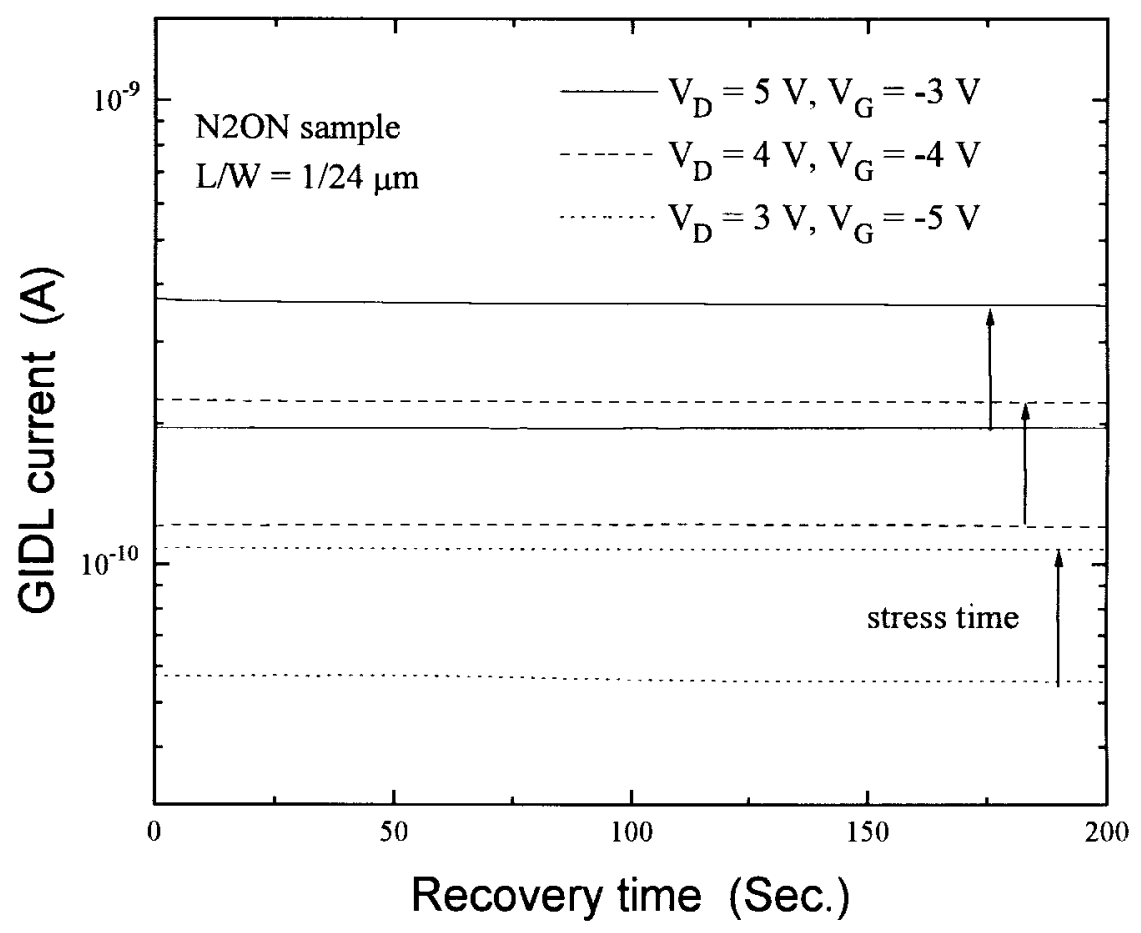

Fig. 3. Measured pre-stress and post-stress GIDL currents for N2ON sample. Stress condition is $V_{G}=0.5 V_{D}=3.5 \mathrm{~V}$ for $3000 \mathrm{~s}$.

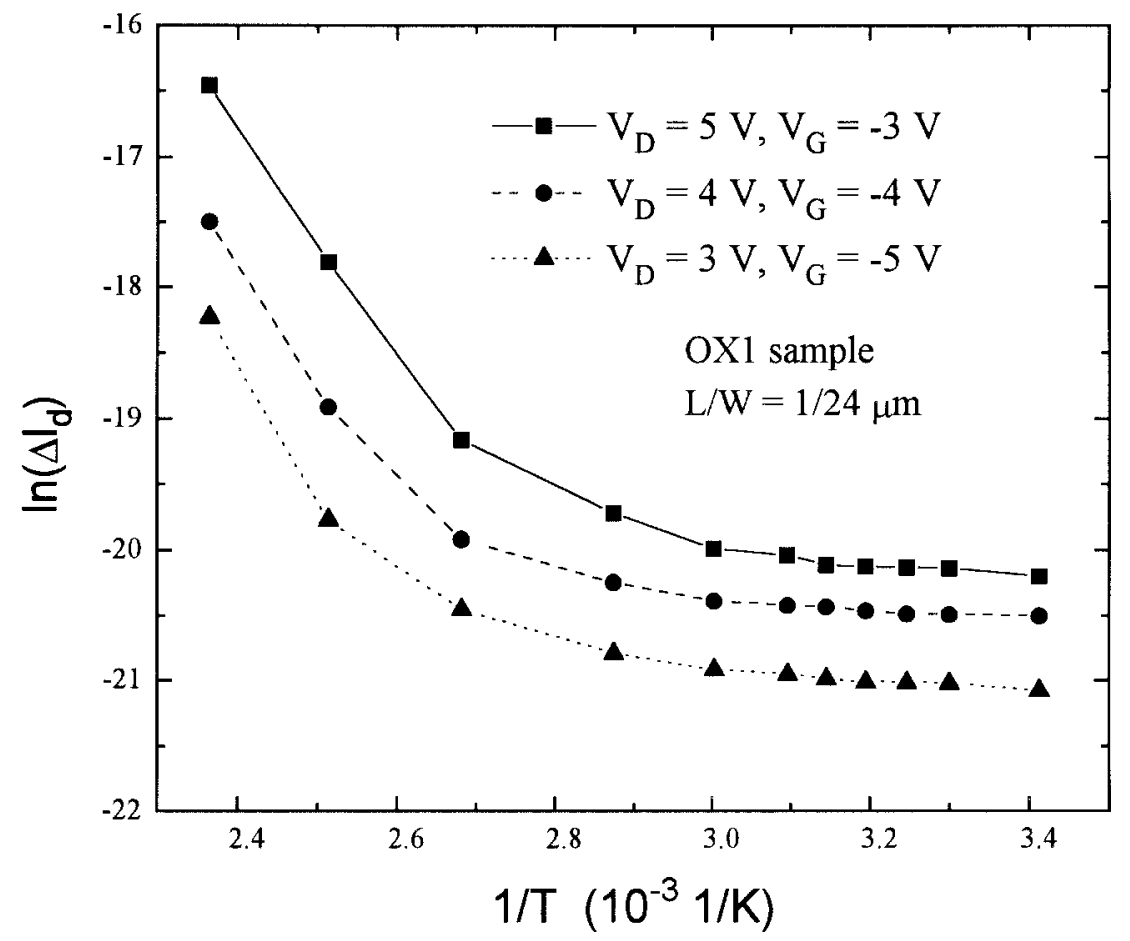

Fig. 4. Temperature dependence of post-stress GIDL increase for OX1 sample. Stress condition is $V_{G}=0.5 V_{D}=3.5 \mathrm{~V}$ for $1000 \mathrm{~s}$.

where $\tau_{0 c}$ is effective transit time in the conduction band, $L_{e}$ is effective spatial width of $\Delta D_{\text {it }}$ distribution which contributes to GIDL current through the two-step tunneling conduction mechanism, $\Delta E_{t}$ is effective energy range covered in GIDL current measurement. In order to deduce $\Delta D_{\mathrm{it}}$, it is necessary to obtain the field distribution near drain junction. Only after $F$ and $F_{1}$ are determined by 2-D device simulation, the value of $B_{\mathrm{it}}$ in (1) can be calculated by (3) and (2), and then $\Delta D_{\mathrm{it}}$ can be found as below.

For clarity, the measured results in Fig. 1 are taken as an example to illustrate the detail of obtaining $\Delta D_{\text {it. }}$. For the three GIDL measurement conditions mentioned previously, the field distributions along the interface near the drain simulated by MINIMOS4 are depicted in Fig. 6. The gate and drain edges 


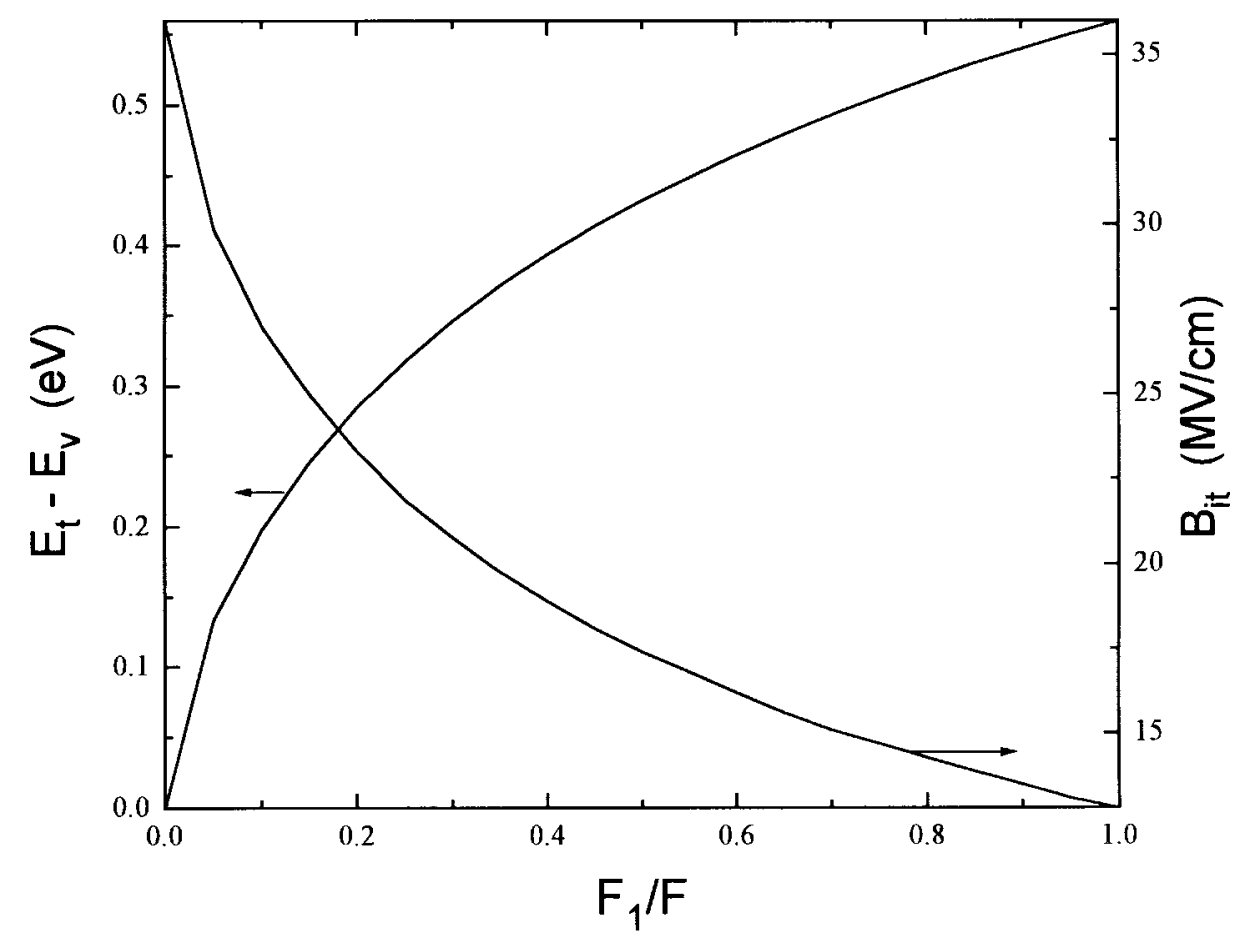

Fig. 5. Changes of trap energy $E_{t}$ and $B_{\text {it }}$ with the ratio of lateral and total fields $F_{1} / F$.

are located at position $x=1.2$ and $1.1 \mu \mathrm{m}$ for OX1 sample; $x=2.0$ and $1.9 \mu \mathrm{m}$ for OX2 sample, respectively, which define the two ends of the gate-drain overlap region. The width of this region was measured by the device-parameter extraction program BSIMPro ${ }^{\circledR}$ for Windows and was equal to $0.10 \mu \mathrm{m}$. From Fig. 6, two phenomena can be observed: the vertical field $F_{2}$ is higher than the lateral field $F_{1}$, and their peaks lie within the gate-drain overlap region. Since $F_{1}$ and $F$ are functions of position, (1) should be rewriten as

$$
\begin{aligned}
\Delta I_{d} & =A \frac{1}{\Delta X} \int \exp \left(-B_{\mathrm{it}} / F\right) d x \\
& \approx A \frac{1}{\Delta X} \sum_{j} \exp \left(-B_{i t, j} / F_{j}\right) \Delta x_{j} .
\end{aligned}
$$

Substituting (4) and re-arranging gives

$$
\Delta D_{\mathrm{it}}=\frac{2 \tau_{0 c} \Delta X}{q W L_{e} \Delta E_{t} \sum_{j} \exp \left(-B_{i t, j} / F_{j}\right) \Delta x_{j}} \cdot \Delta I_{d}
$$

where $\Delta X$ is effective action range of electric field which can result in significant two-step tunneling conduction, and can be approximated to be the same as $L_{e}$. Simulations showed that $\Delta X$ almost corresponds to depletion region width near drain junction which depends on drain voltage, in which depletion region edges in channel region and gate-drain overlap region can be determined by defining a value of $1 \times 10^{14} \mathrm{~cm}^{-3}$ for majority concentration [9]. In addition, from the assumption that $\Delta D_{\mathrm{it}}$ is a uniform distribution only near midgap $E_{i}$ [7], (5,) and (6) should not include the terms with large $B_{i t, j}$ values which correspond to $E_{t}$ levels far below $E i$ as shown in Fig. 5. Calculations showed that when $B_{\text {it }}$ is above $29 \mathrm{MV} / \mathrm{cm}$, electrons tunneling can be almost considered as B-B tunneling because $E_{t}$ is very close to $E_{V}$ in the cases. Combining considerations on $\Delta X$ and $B_{\text {it }}$ above, after performing field simulation, $\Delta D_{\mathrm{it}}$ for different stress times can be calculated using (6) for different $V_{D}$ under same $V_{\mathrm{DG}}$ from the measured $\Delta I_{d}$, and the results are shown in Fig. 7(a) (in the calculation, $\tau_{0 C}=0.1$ ps [10]). For $V_{D}=3,4$, and $5 \mathrm{~V}$ measurement conditions, the values of $\Delta X$ and $\Delta E_{t}$ are $584 \AA, 0.15 \mathrm{eV}$; $1347 \AA, 0.21 \mathrm{eV}$; and $2111 \AA, 0.25 \mathrm{eV}$, respectively, with $\Delta E_{t}$ below $E_{i}$. For comparison, the corresponding results measured by the commonly used $\mathrm{CP}$ method on the same transistor are also presented in Fig. 7(a). As can be seen, the results estimated by GIDL method under all measurement conditions are in good agreement with the results of $\mathrm{CP}$ measurement, and maximum error is less than $6 \%$. The same procedures are also carried out for OX2 sample and results are shown in Fig. 7(b). Two different transistors are used for the two GIDL measurement conditions due to significant oxidecharge trapping effect during GIDL current measurement on the samples, which produces a larger error close to $10 \%$. It is worth pointing out that in Fig. 7(b), the curves for $V_{D}=3$ $\mathrm{V}$ are not included since both measured GIDL current $(<20$ pA) and $\Delta I_{d}$ are too small to be measured accurately, which results in a large error in the calculated $\Delta D_{\text {it }}$ (about an order of magnitude lower than $\mathrm{CP}$ measurement). The fact is also supported by measured results of $\mathrm{N} 2 \mathrm{ON}$ samples $(\mathrm{CP}$ measured value is $9.5 \times 10^{8} \mathrm{~cm}^{-2} \mathrm{eV}^{-1}$ and estimated $\Delta D_{\mathrm{it}}$ is $1.0 \times 10^{9} \mathrm{~cm}^{-2} \mathrm{eV}^{-1}$ for $V_{D}=5 \mathrm{~V}, 8.7 \times 10^{9} \mathrm{~cm}^{-2} \mathrm{eV}^{-1}$ for $V_{D}=4 \mathrm{~V}$, and $6.0 \times 10^{8} \mathrm{~cm}^{-2} \mathrm{eV}^{-1}$ for $V_{D}=3 \mathrm{~V}$ (error: $37 \%$ ). Therefore, to have a properly large measurement current, a suitable drain voltage should be chosen. It has been found in our measurements that GIDL current between hundreds of $\mathrm{pA}$ and tens of $\mathrm{nA}$ is appropriate, whose origin of course must still be examined, i.e., B-B tunneling dominant or 


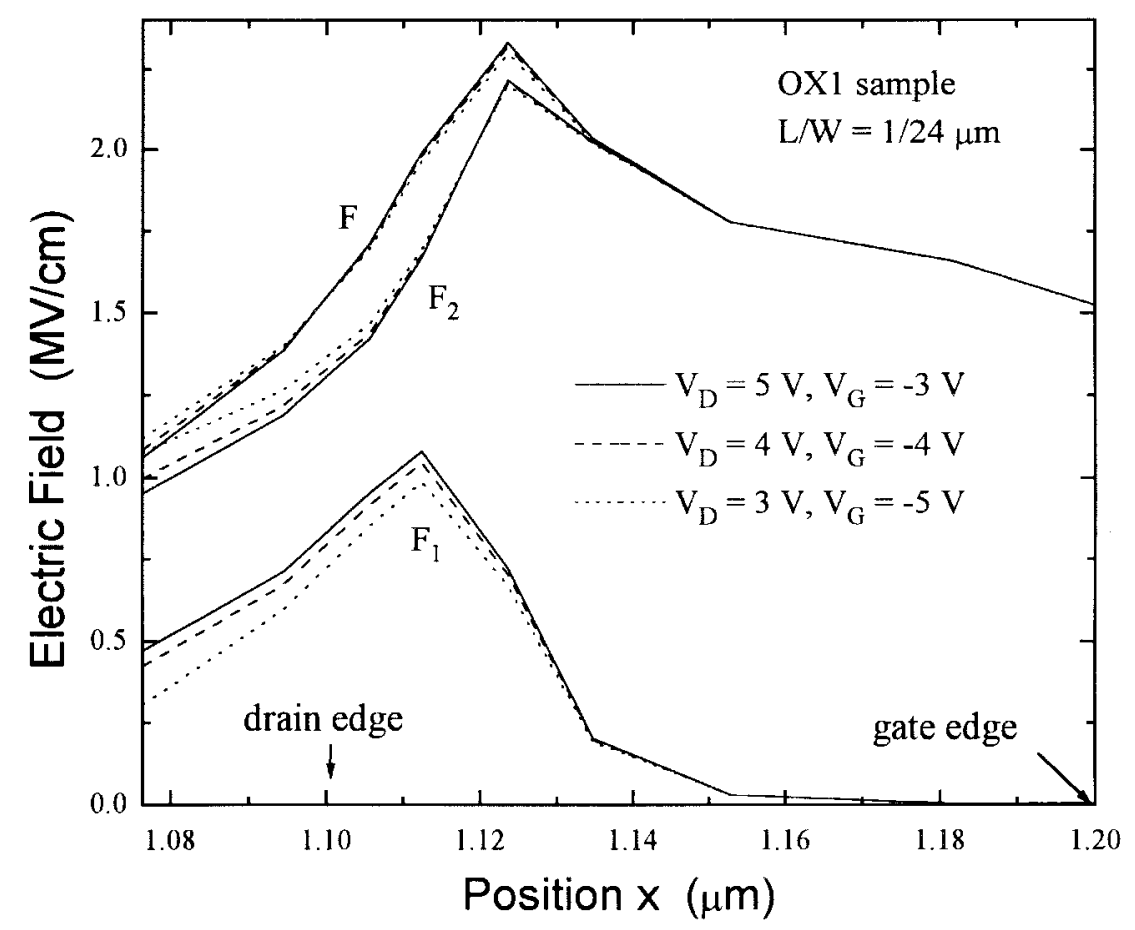

(a)

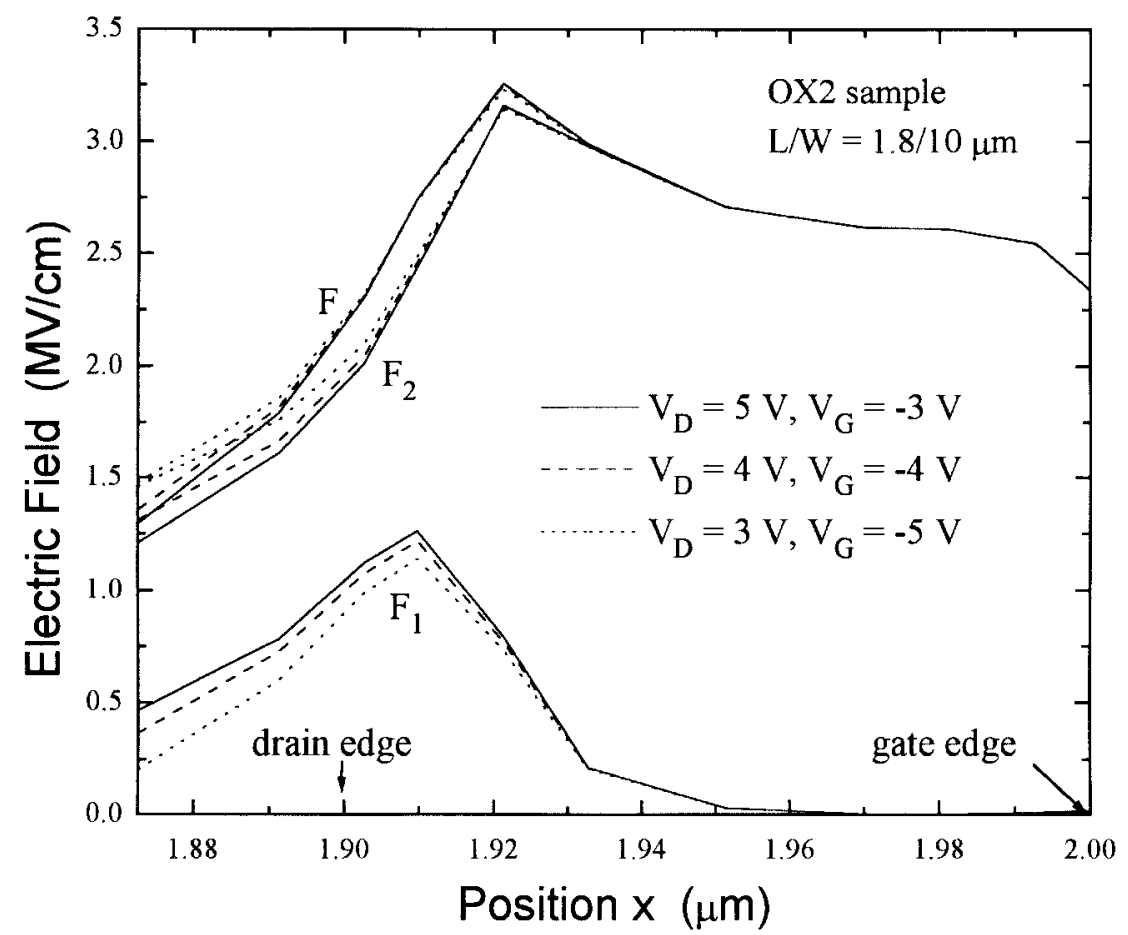

(b)

Fig. 6. Simulated electric field distributions along oxide/Si interface near drain junction for three measurement conditions using MINIMOS4. $V_{S}=V_{B}=0$ V. (a) OX1 sample, and (b) OX2 sample.

two-step tunneling dominant. This can be distinguished simply by plotting $\ln \left(I_{d} / F_{s}\right)$ versus $1 / F_{s}$ for GIDL current resulted from band-to-band tunneling [11]

$$
I_{d}=\frac{C}{B} F_{\text {tot }} \exp \left(-\frac{B}{F_{\text {tot }}}\right)
$$

where $B$ and $C$ are constants, $F_{\text {tot }}$ is the average total electric field which can be approximated by the surface electric field $F_{s}$ at the point of maximum band-to-band tunneling [12]:

$$
F_{s}=-\frac{3 T_{\mathrm{ox}} q N}{\varepsilon_{s}}+\sqrt{\left(\frac{3 T_{\mathrm{ox}} q N}{\varepsilon_{s}}\right)^{2}+\frac{2 q N\left(\left|V_{\mathrm{DG}}\right|-V_{f b}\right)}{\varepsilon_{s}}}
$$

where the drain doping concentration $N$ was simulated to be 


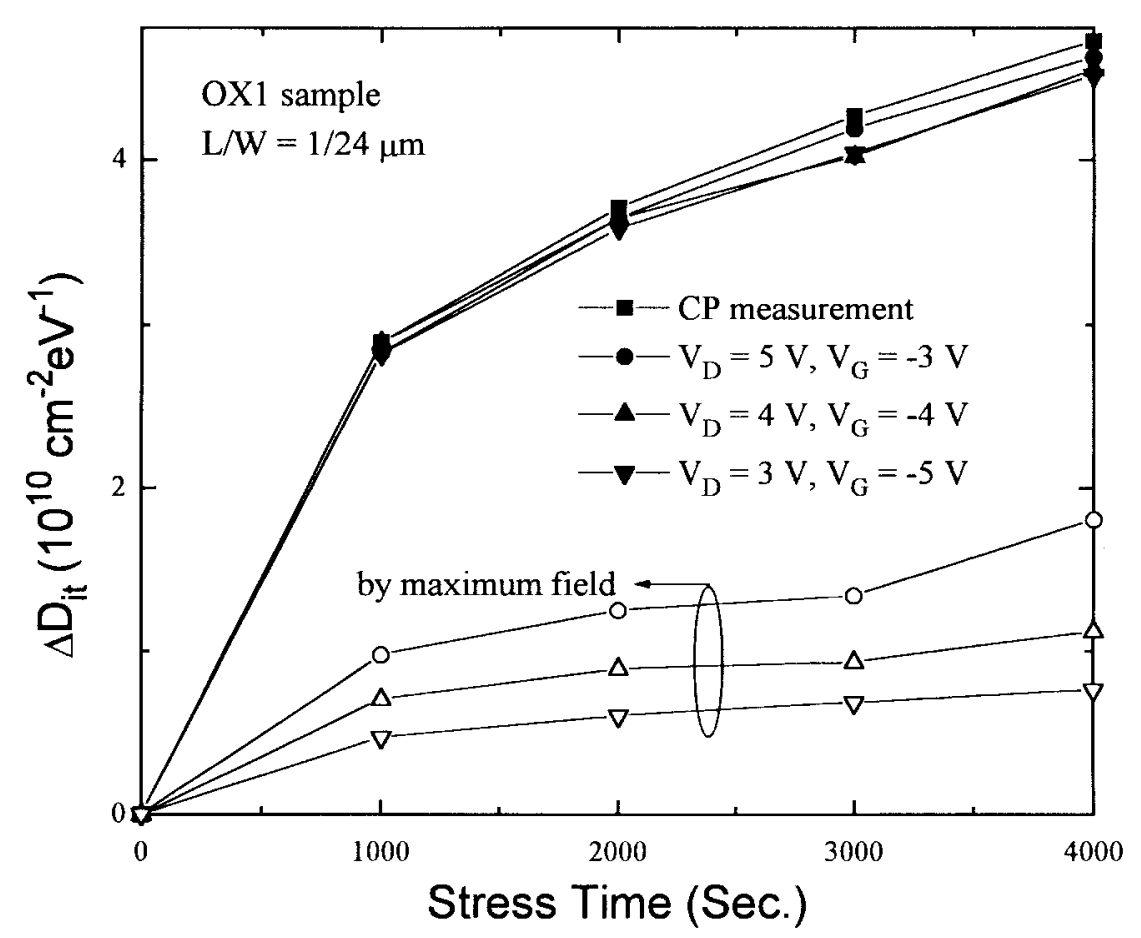

(a)

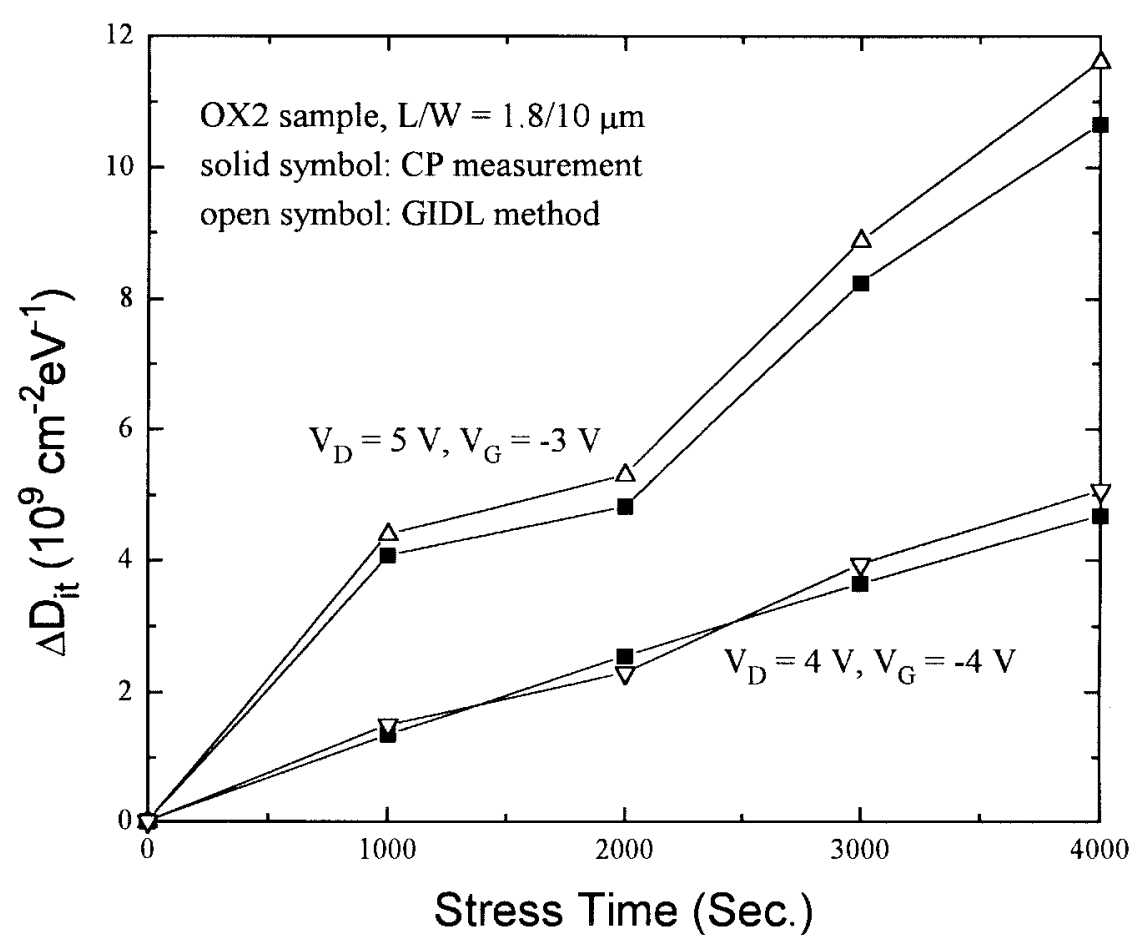

(b)

Fig. 7. $\Delta D_{\text {it }}$ of OX1 (a) and OX2 (b) samples obtained by GIDL current method and CP technique for different stress time. For OX2 sample, measurements for two different $V_{D}$ 's were performed on two different transistors because of oxide-charge trapping during GIDL measurement.

$1.7 \times 10^{19} \mathrm{~cm}^{-3}$, which was verified by spreading-resistance measurements, while flatband voltage $V_{f b}$ was measured to be $-0.866 \mathrm{~V}$ for OX1 sample and $-0.921 \mathrm{~V}$ for OX2 sample. If the $\ln \left(I_{d} / F_{s}\right)$ versus $1 / F_{s}$ curve deviates from a straight line at the chosen $V_{\mathrm{DG}}$, it can be believed that trap-assisted tunneling coexists with B-B tunneling. The two trap-assisted mechanisms can be further distinguished by considering the temperature dependence of $\Delta I_{d}$ shown in Fig. 4. Obviously, in Fig. 8, our measurement condition of $V_{\mathrm{DG}}=8 \mathrm{~V}$ for the GIDL current at room temperature should correspond to the twostep tunneling mechanism because $\Delta I_{d}$ is basically constant around room temperature. 


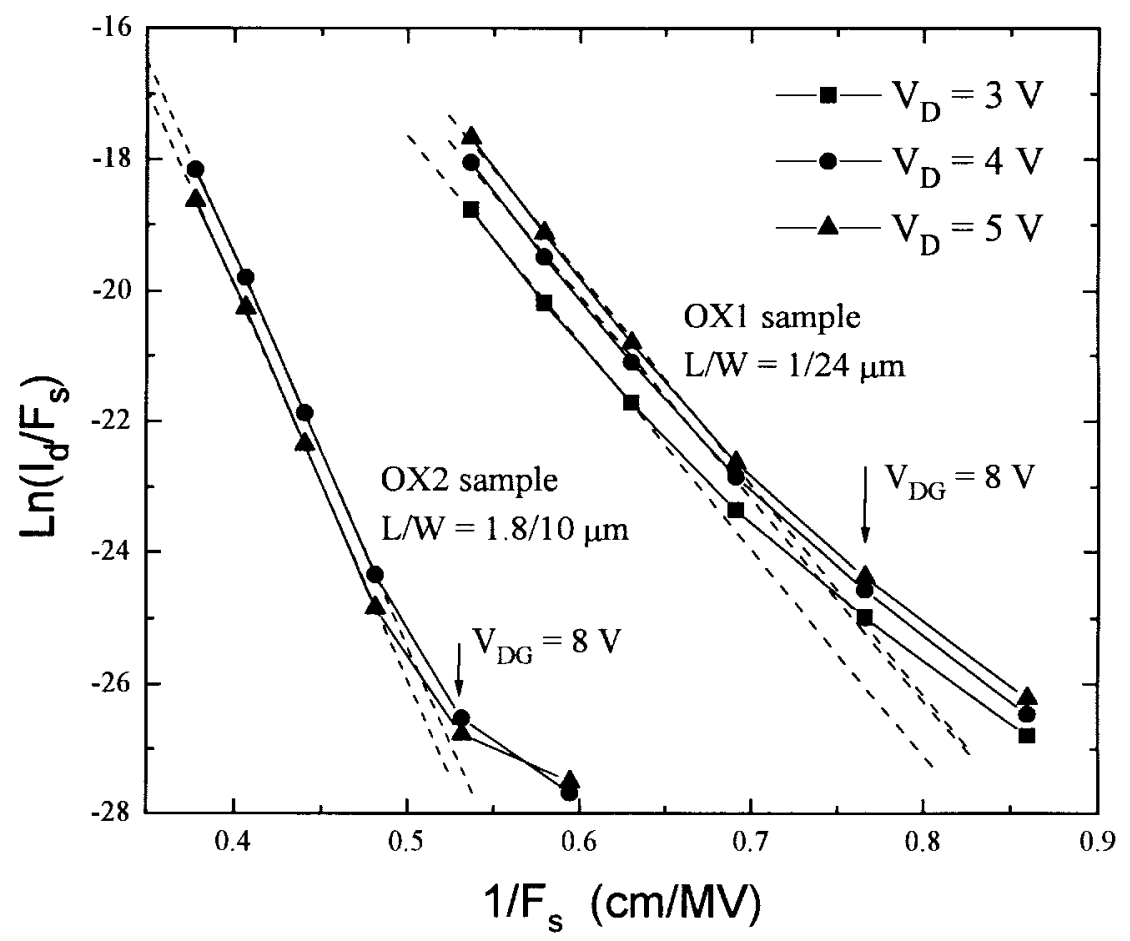

Fig. 8. Plot of $\ln \left(I_{d} / F_{s}\right)$ versus $1 / F_{s}$ for post-stress OX devices. The deviation from linearity at $V_{\mathrm{GD}}=8 \mathrm{~V}$ shows the presence of the two-step tunneling, considering the temperature insensitivity of $\Delta I_{d}$ shown in Fig. 4.

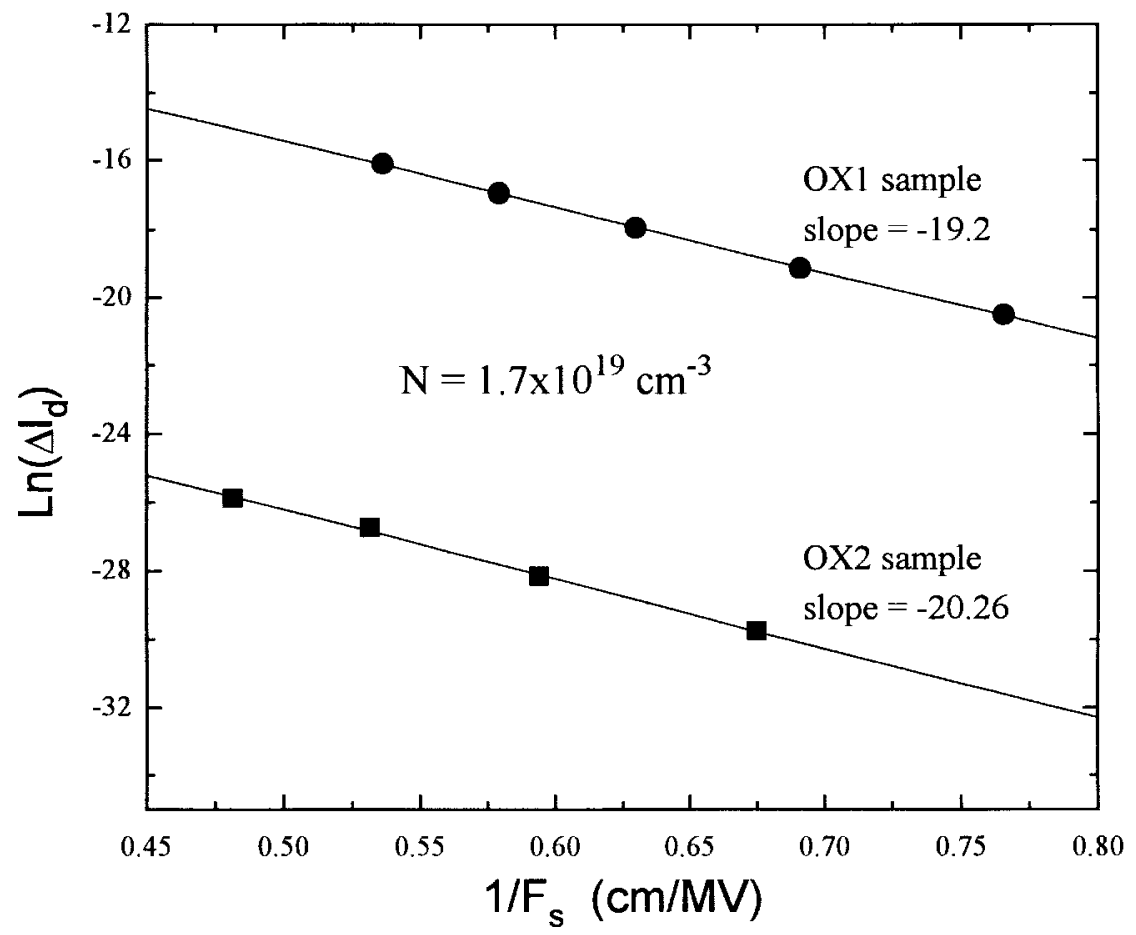

Fig. 9. Plot of $\ln \left(\Delta I_{d}\right)$ versus $1 / F_{s}$ corresponding to the two-step tunneling for OX1 and OX2 samples. From the slope of the linear fit, the values of $B_{\mathrm{it}}, 19.20 \mathrm{MV} / \mathrm{cm}$ for OX1 sample and $20.26 \mathrm{MV} / \mathrm{cm}$ for OX2 sample, are obtained.

It should be emphasized that if (1) is adopted with $F$ taking the maximum value of the field distribution, the estimated $\Delta D_{\mathrm{it}}$ is much smaller than that by CP method (error $\sim 68 \%$ for $V_{D}=5 \mathrm{~V}, 78 \%$ for $V_{D}=4 \mathrm{~V}$, and $84 \%$ for $V_{D}=3$ V) as shown in Fig. 7(a), while one using the average field in (8) instead is several orders larger than that by CP method [not shown in Fig. 7(a)]. This indicates that the whole field distribution near the drain junction is needed in order to accurately link the GIDL increase with $\Delta D_{\text {it }}$. Finally, the average value of $B_{\mathrm{it}}$ is directly found by plotting $\ln \left(\Delta I_{d}\right)$ versus $1 / F_{s}$. As shown in Fig. 9 slope $=-19.20$ for OX1 sample, -20.26 for OX2 sample, i.e., $B_{\mathrm{it}}$ of OX1 and OX2 samples is 19.20 and $20.26 \mathrm{MV} / \mathrm{cm}$, respectively, which are in good agreement with the average values of $19.45 \mathrm{MV} / \mathrm{cm}$ for 
OX1 sample and $20.22 \mathrm{MV} / \mathrm{cm}$ for OX2 sample obtained by simulation of the field distribution, suggesting good accuracy of the field simulation.

\section{SUMMARY}

In summary, the dependence of post-stress GIDL current increase $\Delta I_{d}$ on the creation of interface states during hot-carrier stress was investigated by means of a two-step tunneling model. The increase can reflect the change of interface-state density $\Delta D_{\mathrm{it}}$ under proper drain-gate biases. The two-step tunneling results in a $\Delta I_{d}$ insensitive to temperatures up to about $50{ }^{\circ} \mathrm{C}$. For a fixed $V_{\mathrm{DG}}$ of $8 \mathrm{~V}$ in our devices, the energy level of the traps which is effective for the twostep tunneling is within a range of $0.15-0.25 \mathrm{eV}$ below the midgap and the width of the tunneling region is about 600-2000 $\AA$ near the drain junction for $V_{D}$ of 3-5 V. From the developed relation between $\Delta D_{\mathrm{it}}$ and $\Delta I_{d}, \Delta D_{\mathrm{it}}$ can be estimated from the measured $\Delta I_{d}$ and 2-D device simulation, and the maximum error is smaller than $10 \%$ relative to the results of charge-pumping measurement. Further support is obtained by using devices with nitrided gate oxide (which show much suppressed $\Delta D_{\mathrm{it}}$ ), different gate-oxide thicknesses and different channel dimensions. Therefore, this work is conducive to understanding some properties of $\Delta D_{\mathrm{it}}$ and its relation with $\Delta I_{d}$.

\section{REFERENCES}

[1] B. Doyle, M. Bourcerie, J. C. Marchetaux, and A. Boudou, "Interface state creation and charge trapping in the medium-to-high gate voltage range during hot-carrier stressing of n-MOS transistors," IEEE Trans. Electron Devices, vol. 37, pp. 744-754, Mar. 1990.

[2] T. Wang, T. E. Chang, C. M. Huang, J. Y. Yang, K. M. Chang, and L. P. Chiang, "Structural effect on band-trap-band tunneling induced drain leakage in n-MOSFET's," IEEE Electron Device Lett., vol. 16, pp. 566-568, Dec. 1995.

[3] G. Q. Lo, A. B. Joshi, and Dim-Lee Kwong, "Hot-carrier-stress effect on gate-induced drain leakage current in n-channel MOSFET's," IEEE Electron Device Lett., vol. 12, pp. 5-7, Jan. 1991.

[4] C. Duvvury, D. J. Redwine, and H. J. Stiegler, "Leakage current degradation in N-MOSFET's due to hot-electron stress," IEEE Electron Device Lett., vol. 9, pp. 579-581, Nov. 1988.

[5] I. C. Chen, C. W. Teng, D. J. Coleman, and A. Nishimura, "Interfacetrap enhanced gate-induced leakage current in MOSFET," IEEE Electron Device Lett., vol. 10, pp. 216-218, May 1989.

[6] T. Hori, "Drain-structure design for reduced band-to-band and band-todefect tunneling leakage," in 1990 Symp. VLSI Technol., pp. 69-70.

[7] T. Wang, E. Chang and C. Huang, "Interface trap induced thermionic and field emission current in off-state MOSFET's," in IEDM Tech. Dig., 1994, pp. 161-164.

[8] X. Zeng, P. T. Lai, and W. T. Ng, "A novel technique of $\mathrm{N}_{2} \mathrm{O}$-treatment on $\mathrm{NH}_{3}$-nitrided oxide as gate dielectric for nMOS transistors," IEEE Trans. Electron Devices, vol. 43, pp. 1907-1913, Nov. 1996.

[9] W. Chen, B. Artur, and Tso-Ping Ma, "Lateral profiling of oxide charge and interface traps near MOSFET junctions," IEEE Trans. Electron Devices, vol. 40, pp. 187-196, Jan. 1993.

[10] A. W. De Groot, G. C. McGonigal, D. J. Thomson, and H. C. Card, "Thermionic-field emission from interface states at grain boundaries in silicon," J. Appl. Phys., vol. 55, pp. 312-317, 1984.

[11] H. J. Wann, P. K. Ko, and C. Hu, "Gate-induced band-to-band tunneling leakage current in LDD MOSFET's," in IEDM Tech. Dig., 1992, pp. 147-150.

[12] S. A. Parke, J. E. Moon, H. J. Wann, P. K. Ko, and C. Hu, "Design for suppression of gate-induced drain leakage in LDD MOSFET's using a quasi-two-dimensional analytical model," IEEE Trans. Electron Devices, vol. 39, pp. 1694-1703, July 1992.

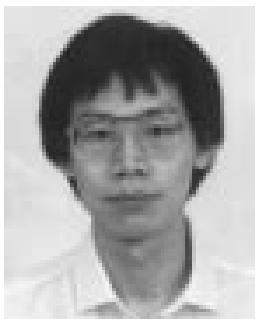

P. T. Lai received the Ph.D. degree from the University of Hong Kong in 1985. His research was related to the design of small-sized MOS transistors with emphasis on the narrow-channel effects. The work involved analytical and numerical modelings, and different isolation structures.

He worked as a Post-Doctoral Fellow at the University of Toronto, Toronto, Ont., Canada, in the area of self-aligned bipolar transistor using a poly-emitter bipolar process with trench isolation. Currently, he is with the University of Hong Kong, where his current research interests include investigation of various physical mechanisms that govern the complexity of IC's, development of efficient algorithms and models for the simulations of IC process and semiconductor devices, the development of a PC-based CAD tool for IC technologies, covering process, device and circuit levels, and integrated sensors.

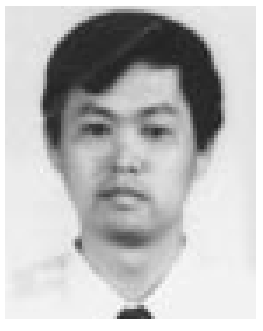

J. P. Xu received the B.S., M.S. and Ph.D. degrees in microeletronics from Huazhong University of Science and Technology, Wuhan, P.R.C., in 1982, 1984, and 1993, respectively.

He is currently a Post-Doctoral Fellow in the Department of Electrical and Electronic Engineering, the University of Hong Kong. His current research interests include the reliability of MOSFET's and the performance of MOSFET's with NO-based, $\mathrm{N}_{2} \mathrm{O}$-based, and $\mathrm{NH}_{3}$-based nitrided oxide as gate dielectrics.

W. M. Wong is currently a student in the Department of Electrical and Computer Engineering at the University of Calgary, Calgary, Alta., Canada.

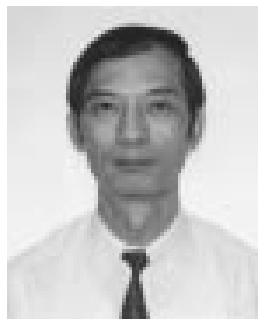

H. B. Lo received the B.Eng. and M.Eng. degrees in engineering physics from McMaster University, Hamilton, Ont., Canada, in 1970 and 1972, respectively.

From 1970 to 1972, he was a Research Engineer in the Atomic Power Division and the Solid State Devices Department at Westinghouse Canada, Hamilton. From 1974 to 1980, he was with Micro Electronics, Ltd., Hong Kong, where he was engaged in both development and production of wafer processing of bipolar devices. In 1980, he joined the Department of Electrical and Electronic Engineering, the University of Hong Kong, where he is currently a Lecturer. His current research interests are on the design and processing of semiconductor devices.

Y. C. Cheng (M'78) received the B.Sc. degree in physics and mathematics from the University of Hong Kong in 1963 and the Ph.D. degree in theoretical physics from the University of British Columbia, Vancouver, B.C., Canada, in 1967.

From 1963 to 1978 , he held various appointments at a number of universities in Canada and at Bell-Northern and Xerox Research Laboratories. During this period, he had undertaken research work on silicon devices and had coinvented and developed the "HCI-oxidation" technique for the production of clean oxides for integrated-circuit applications. In 1977, he was appointed as an Adjunct Professor at the University of Waterloo, Waterloo, Ont., Canada. He was a Professor in the Department of Electrical and Electronic Engineering and Dean of the Engineering Faculty, University of Hong Kong. In 1986, he was appointed as a Visiting Professor in EECS, University of California, Berkeley. He is also an Advisory Professor of the Physics Department, the South China University of Technology. He has authored or coauthored over 100 technical papers and holds three U.S. and Canadian patents on semiconductor technology. Currently, he is the Vice Chancellor of the University of Hong Kong. 